\title{
Effectiveness of training methods for delivery of evidence-based psychotherapies: a systematic review
}

\author{
Helen Valenstein-Mah ${ }^{1 *}$ D, Nancy Greer ${ }^{2,3}$, Lauren McKenzie ${ }^{2,3}$, Lucas Hansen ${ }^{3,4}$, Thad Q. Strom5,
} Shannon Wiltsey Stirman ${ }^{6,7}$, Timothy J. Wilt ${ }^{2,3,8}$ and Shannon M. Kehle-Forbes 2,3,8,9 $^{2}$

\begin{abstract}
Background: Extensive efforts have been made to train mental health providers in evidence-based psychotherapies (EBPs); there is increasing attention focused on the methods through which providers are trained to deliver EBPs. Evaluating EBP training methods is an important step in determining which methods are most effective in increasing provider skill and improving client outcomes.

Methods: We searched MEDLINE (Ovid) and PsycINFO for randomized controlled trials published from 1990 through June 2019 that evaluated EBP training methods to determine the effectiveness of EBP training modalities on implementation (provider and cost) and client outcomes. Eligible studies $(N=28)$ were evaluated for risk of bias, and the overall strength of evidence was assessed for each outcome. Data was extracted by a single investigator and confirmed by a second; risk of bias and strength of evidence were independently rated by two investigators and determined by consensus.

Results: Overall, EBP training improved short-term provider satisfaction, EBP knowledge, and adherence compared to no training or self-study of training materials (low to moderate strength of evidence). Training in an EBP did not increase treatment adoption compared to no training or self-study. No specific active EBP training modality was found to consistently increase provider EBP knowledge, skill acquisition/adherence, competence, adoption, or satisfaction compared to another active training modality. Findings were mixed regarding the additive benefit of post-training consultation on these outcomes. No studies evaluated changes in provider outcomes with regards to training costs and few studies reported on client outcomes.

Limitations: The majority of included studies had a moderate risk of bias and strength of evidence for the outcomes of interest was generally low or insufficient. Few studies reported effect sizes. The ability to identify the most effective EBP training methods was limited by low strength of evidence for the outcomes of interest and substantial heterogeneity among studies.

(Continued on next page)
\end{abstract}

* Correspondence: hvalmah@umn.edu

'Department of Psychiatry and Behavioral Sciences, University of Minnesota

Medical School, 2450 Riverside Avenue, F282/2A West, Minneapolis, MN

55454, USA

Full list of author information is available at the end of the article

(c) The Author(s). 2020 Open Access This article is licensed under a Creative Commons Attribution 4.0 International License, which permits use, sharing, adaptation, distribution and reproduction in any medium or format, as long as you give appropriate credit to the original author(s) and the source, provide a link to the Creative Commons licence, and indicate if changes were made. The images or other third party material in this article are included in the article's Creative Commons licence, unless indicated otherwise in a credit line to the material. If material is not included in the article's Creative Commons licence and your intended use is not permitted by statutory regulation or exceeds the permitted use, you will need to obtain permission directly from the copyright holder. To view a copy of this licence, visit http://creativecommons.org/licenses/by/4.0/ The Creative Commons Public Domain Dedication waiver (http://creativecommons.org/publicdomain/zero/1.0/) applies to the data made available in this article, unless otherwise stated in a credit line to the data. 
(Continued from previous page)

Conclusions: EBP training may have increased short-term provider satisfaction, EBP knowledge, and adherence though not adoption. Evidence was insufficient on training costs and client outcomes. Future research is needed on EBP training methods, implementation, sustainability, client outcomes, and costs to ensure efforts to train providers in EBPs are effective, efficient, and durable.

Trial registration: The protocol for this review is registered in PROSPERO (CRD42018093381).

Keywords: Evidence-based psychotherapy, Provider training, Training methods, Adherence, Competence, Fidelity

\section{Contributions to the literature}

- Training in evidence-based psychotherapies (EBPs) probably increased short-term provider satisfaction, treatment knowledge, and adherence. Training in an EBP may not have increased EBP adoption compared to no training or self-study.

- No active training modality demonstrated clear superiority over another for provider implementation outcomes. Findings were mixed regarding the additive benefit of posttraining consultation on these outcomes. While data was limited, costs of training methods were highest with in-person training and lowest with online training

- Strength of evidence for outcomes was generally low or insufficient. Future research should focus on training costs and client outcomes as well as sustainability of training effects.

\section{Background}

Extensive efforts have been made to train mental health providers in evidence-based psychotherapies (EBPs) treatments that have been empirically evaluated and have demonstrated effectiveness in controlled research studies [1]. Substantial financial and organizational resources have been put toward disseminating and implementing EBPs through nationally funded agencies such as the Substance Abuse and Mental Health Services Administration (SAMHSA) and the National Child Traumatic Stress Network (NCTSN), state-funded initiatives, and public healthcare systems such as the Veterans Health Administration (VHA) [2, 3]. In community settings, legislators have passed policies mandating that evidence-based practices be implemented in public mental healthcare $[4,5]$. One institutional exemplar in training providers in EBPs is the VHA, the largest integrated healthcare system in the USA. VHA has developed a handbook with expectations and procedures for implementing EBPs at Veterans Affairs (VA) institutions [6] and has trained over 11,600 mental health staff in at least one EBP since 2007 [7]. Taken together, these diverse efforts to implement EBPs highlight both the need, and rapidly growing demand, for access to effective mental health treatment.
Alongside efforts to implement EBPs, there is increasing attention focused on the methods through which providers are trained to deliver EBPs. Evaluating EBP training and determining "evidence-based training" methods is of considerable importance [8]. While earlier research is mixed [9], recent evidence suggests that the fidelity with which EBPs are delivered affects client outcomes, emphasizing the need for well-trained providers $[10,11]$. Conversely, ineffective training that does not enhance providers' EBP skill and competency could result in poorer health outcomes than those reported in controlled trials demonstrating EBP efficacy.

The effectiveness of EBP training may be evaluated in the context of different implementation outcomes. Proctor and colleagues (2011) identify three distinct, although interrelated, outcome domains: implementation, service, and client outcomes [12]. Provider-level outcomes typically targeted through EBP training can be considered under the umbrella of implementation outcomes and include (a) satisfaction (acceptability of the training to providers), (b) adoption (use of EBP by a provider post-training), (c) knowledge (understanding of EBP principles and techniques), (d) skill acquisition or adherence (ability to employ core techniques/interventions of an EBP), (e) competence (skill with which EBP techniques are delivered), and (f) fidelity (composite of adherence and competence to EBP) [9, 13]. Additional outcomes at the implementation-level include training costs such as financial resources and provider time, including lost productivity and client care hours due to time spent in training activities. Service outcomes, derived from the Institute of Medicine's quality improvement aims, were not directly examined in this review. Finally, client outcomes include symptom reduction, functional improvement, and treatment satisfaction (see Fig. 1 for conceptual model).

The use of different implementation strategies [14] may lead to more or less successful implementation outcomes. Some EBP training methods, such as selfdirected study of treatment materials and in-person workshops without follow-up, do not increase provider skill acquisition [15, 16]. More intensive training methods may lead to meaningful gains in provider skill in delivering an EBP. For example, an earlier review 


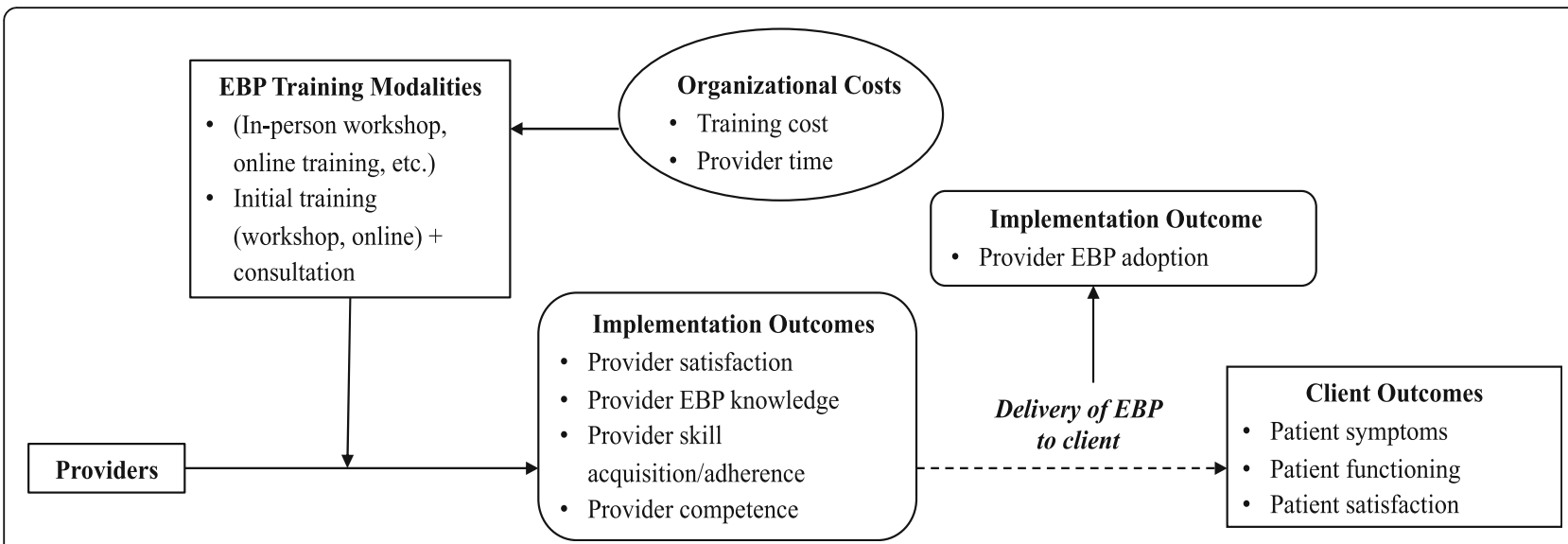

Fig. 1 Analytic framework for evaluation of evidence-based psychotherapy (EBP) training methods based on Proctor et al. (2011) conceptual framework for implementation outcomes

found evidence that workshops with additional followup and "multi-component" trainings that included observation, feedback, consultation, and coaching increased skill acquisition [15]. However, it remains unclear if more rigorous training methods that may improve provider outcomes also improve client outcomes. Additionally, more intensive training methods can be resource-intensive and limit EBP spread. These limitations in resources have led to development of efficient, scalable, and lower cost training methods such as online training and blended learning models, which incorporate elements of both online training and expert consultation. Such an approach may increase provider access [17], especially where expert training is limited, such as in rural areas and developing countries.

To date, the ability to reach conclusions regarding the most effective EBP training methods has been hampered by methodological limitations (e.g., pre-post, non-controlled studies) and the use of self-assessment of provider skill acquisition and competence, which is not always consistent with objective measurement of provider behavior [15]. Moreover, studies on EBP training have not routinely reported client outcomes, and most research has focused exclusively on mental health providers, although it is becoming increasingly common for non-mental health professionals (e.g., primary care providers, nurses) to deliver brief EBPs for mental health conditions such as alcohol misuse [18]. Evaluating the evidence for EBP training methods is an important step in determining the effectiveness and comparative effectiveness on implementation, system, and client outcomes, with the goal of increasing provider skill, improving client outcomes, and containing training costs. Finally, while the implicit goal of EBP training has been to increase the reach of these treatments, the psychotherapy training literature has largely developed in parallel to implementation science. Historically, EBP training and clinical supervision has focused on building providers' expertise in the EBP and has lacked implementation strategies to integrate and sustain the clinical innovation. Mapping strategies used in EBP training and consultation/ supervision and defined implementation strategies [14] will facilitate comparisons across clinical innovations and identify future EBP training research directions.

\section{Objectives}

We conducted a systematic review of randomized controlled trials that evaluated EBP training methods to determine the effectiveness and comparative effectiveness of EBP training modalities (e.g., in-person training with consultation, blended-learning, online only) on implementation outcomes (e.g., provider EBP knowledge, adherence, competence, and fidelity as well as costs such as financial resources and provider time). We also examined the effect of EBP training on client outcomes (e.g., symptom scores).

\section{Methods}

Details of the methods for our systematic review are described below and correspond with the Preferred Reporting Items for Systematic Reviews and Meta-Analyses (PRISMA) guidelines (see supplemental material for PRISMA checklist) [19]. The protocol for this review is registered in the international prospective register of systematic reviews (PROSPERO; CRD42018093381).

\section{Searches}

We searched MEDLINE (Ovid) and PsycINFO for studies published from 1990 through June 2019. See Table 1 for the full electronic search strategy used for MEDLINE. We hand-searched the reference lists of relevant systematic reviews to identify additional studies that may have been missed in our electronic search.

\section{Study inclusion and exclusion criteria}

Studies were potentially eligible for inclusion if they evaluated methods used to train providers to deliver 
Table 1 MEDLINE (Ovid) search strategy

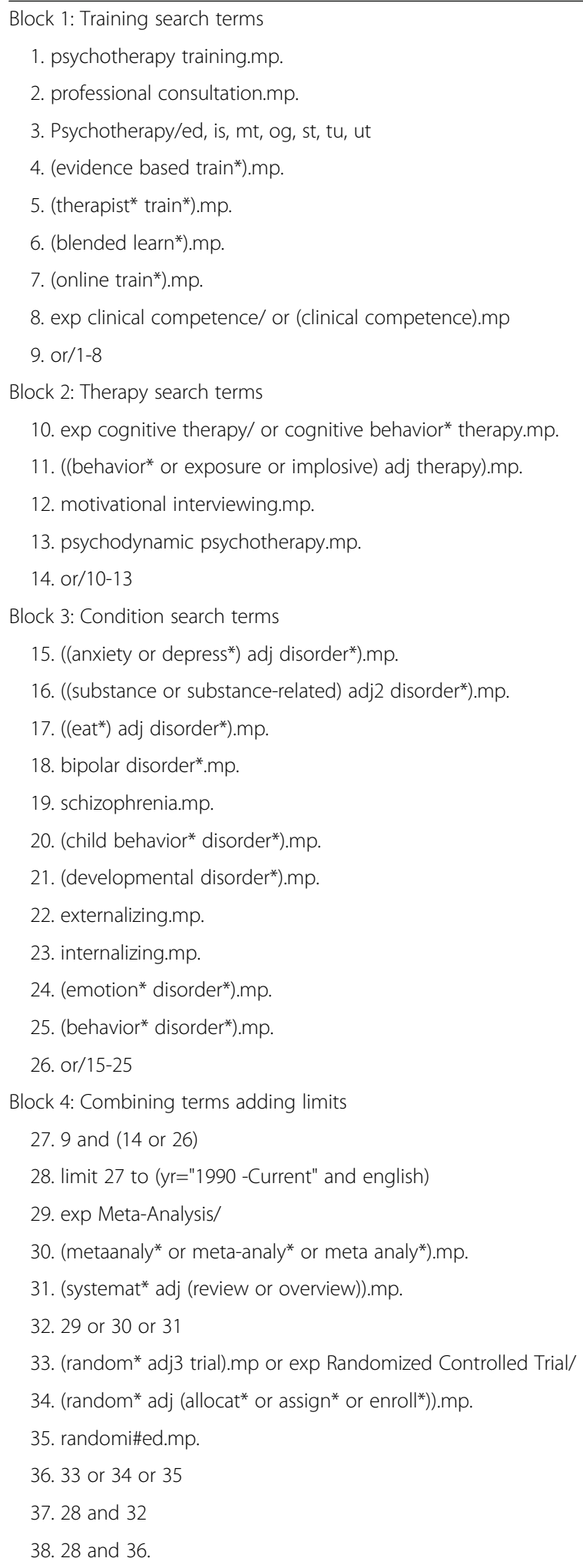

EBPs to individuals with mental health diagnoses. We included randomized controlled studies if (1) they were published in a peer reviewed journal, in English, between 1990 and June 2019 (date range was in line with Herschell and colleagues' (2010) review as prior reviews summarized previous research [20], and EBPs were still largely in development at this time); (2) the intervention that providers were trained in was an EBP (determined through consensus by the first and last authors), using available guides [21, 22]; (3) EBPs were designed to treat individuals who had a mental health condition; (4) authors included information on the EBP training (e.g., method used and components of training); and (5) relevant training outcomes were reported (see provider-level outcomes below). We excluded studies that did not evaluate provider training in the EBP. Study inclusion criteria was deliberately kept broad with respect to provider characteristics/training, EBPs which were the focus of trainings, and client mental health conditions in order to maximize the number of relevant studies given the relatively small body of controlled research on training methods to date.

Studies must have reported at least one of the following provider-centered outcomes to be eligible for the review: (a) provider satisfaction with training, (b) provider treatment knowledge, (c) provider skill acquisition, (d) provider adherence; (e) provider competence, (f) provider fidelity, or (g) EBP adoption (e.g., use of EBP after the training was completed). Additionally, the following outcomes were examined if reported: (a) training cost and (b) client outcomes post-EBP training. For included studies, the provider population (e.g., individuals receiving the EBP training) could include any health professional (e.g., psychologist, social worker, primary care physician, addiction specialist, etc.) delivering an EBP. If clients were included in the study, the client population (e.g., individuals receiving the EBP) was defined as individuals receiving the mental health treatment by study providers. All EBP training modalities (e.g., workshops, online training) were eligible, including studies that manipulated post-initial training consultation. EBP training modalities could be compared to other active training conditions, training controls, or waitlist conditions, and they needed to assess provider outcomes at least preand post-training. Additional follow-up was not required for inclusion.

\section{Study quality assessment}

We assessed risk of bias for individual studies included in the review using a modification of the Cochrane risk of bias criteria [23]. The risk of bias rating was based on the following categories: random sequence generation, allocation concealment, masking of participants and/or personnel, attrition bias, and 
selective outcome reporting. Categories which were not described adequately in the paper were given an "unclear" designation (considered a medium risk of bias). Given the nature of the studies included in the review (e.g., studies in which providers are trained in EBPs), we considered it difficult for studies to mask participants to training condition. Thus, studies could still qualify as having a low risk of bias rating for masking if personnel involved in outcome assessment (e.g., simulated clients; adherence coders) were masked to the participants' condition. Regarding attrition bias, we categorized studies as having low risk of bias if they had less than $10 \%$ incomplete provider outcome data due to attrition, medium if they had between 10 and 20\%, and high if they had more than $20 \%$. For overall risk of bias, studies were categorized as low if they were rated as low for random sequence generation and masking, low or medium for attrition, and had no categories rated as high. Studies were rated as having overall medium risk of bias if they had no more than one category rated as high, and studies were rated as having an overall high risk of bias if they had more than one category rated as high.

Finally, the overall strength of evidence for each outcome was evaluated using the five required domains outlined in the Agency for Healthcare Research and Quality rating system [24]. Specifically, for each outcome, the quality of evidence rating was based on studies' limitations (risk of bias), consistency in direction/magnitude of effects, directness (interventions relation to clinical health outcome), precision (degree of certainty regarding effect estimate), and potential reporting bias. The overall strength of the evidence was then rated as high, moderate, low, or insufficient based on aggregation of these five domain ratings. Risk of bias and quality of evidence were rated by two independent reviewers and inconsistencies in ratings were discussed to reach consensus.

\section{Data extraction strategy}

Two trained reviewers independently reviewed all study abstracts to identify potentially eligible articles based on the a priori criteria listed above. Studies underwent fulltext review if either reviewer thought it was relevant to the review's aims and appeared to meet inclusion criteria. The studies selected for full-text review were again evaluated independently by two reviewers. Studies were included in the systematic review if both investigators deemed them to meet the inclusion criteria. Investigators discussed any discrepancies regarding their inclusion/exclusion decision and consulted with a third member of the review team if necessary.

\section{Extraction of data items}

Data extraction was completed by one reviewer and verified by a second. We extracted the following elements from each study:
1. Study characteristics including training methods evaluated, EBP type and mental health condition, provider and client characteristics, assessment measures, and assessment timeframe

2. Provider outcomes including satisfaction with training, treatment knowledge, skill acquisition, adherence, competence, fidelity, and adoption

3. Cost of training

4. Client clinical outcomes (e.g., symptom scores)

We also extracted the components (e.g., role-play, expert consultation, video/audio review) of each training method evaluated in the studies included in the review.

\section{Data synthesis and presentation}

We generated descriptive statistics (i.e., count and percent) of articles reporting on each EBP training modality as well as each provider-centered outcome. Given the heterogeneity of the training methods, assessment measures, and providers and clients, we described the results using a narrative/descriptive synthesis instead of a quantitative analysis.

\section{Results}

The literature flow is reported in Fig. 2. Our electronic and hand searches yielded a total of 1844 studies. After removal of duplicates $(n=355), 1489$ studies underwent abstract review. Of those studies, 1368 studies were subsequently excluded, leaving 121 articles for full-text review, including nine studies identified through a hand search of recent systematic reviews and reference lists of included studies. Of those, 93 were excluded for a total of 28 eligible and included studies [25-52].

Table 2 includes a description of the characteristics of included studies and Table 3 characterizes discrete implementation strategies used in the EBP training methods included in each study. Additional study information and risk of bias for each study are reported on Appendix Table 1. Overall, there was considerable heterogeneity between studies. Three quarters (75\%) of studies were conducted in the USA, with the remainder in multi-country or international settings. Samples sizes of providers ranged from $n=30$ to $n=181$, with $39 \%$ of studies having over 100 providers and 32\% having less than 50 providers. Provider characteristics (e.g., degree, previous experience with EBP; Appendix Table 1) also varied widely between studies and $50 \%$ of the studies included providers who were unlicensed/trainees. Slightly over half $(57.1 \%)$ of studies included an objective assessment of provider behavior (e.g., rating of provider's adherence in a simulated client interaction); the remainder of the studies (with the exception of one which reported on client outcomes only) used an EBP knowledge test and/or client report/provider self-report of provider 


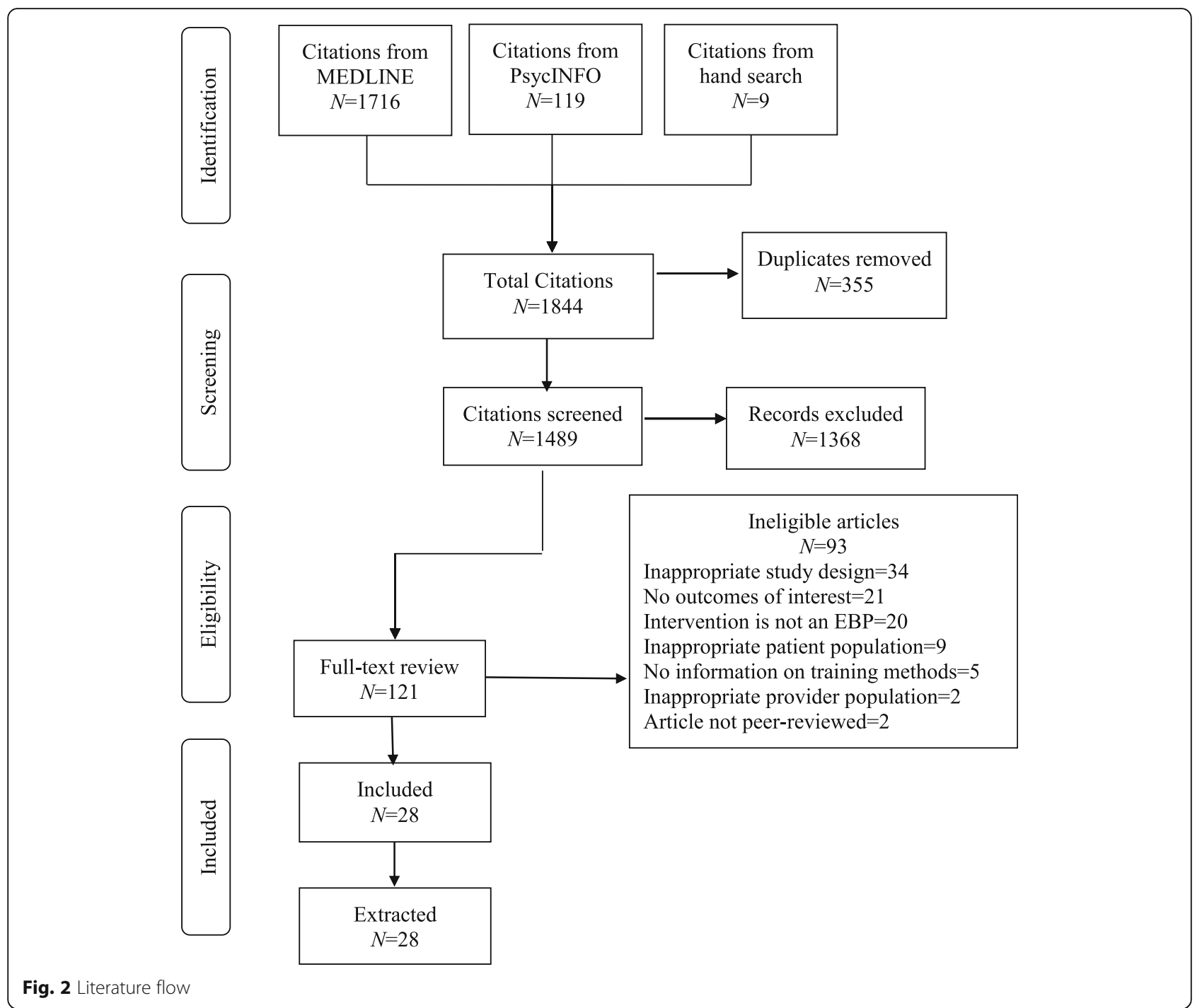

behavior to assess the effects of training. The definition and measurement of provider outcomes varied between studies (Appendix Table 1) and follow-up timeframe for assessment of provider outcomes ranged between studies from pre-training to immediately post-training only to pre-training to 12-months post-training. Regarding type of EBP, $36 \%$ of studies evaluated training for a variant of Cognitive Behavioral Therapy (e.g., a range of Cognitive Behavioral Therapy protocols designed to treat different mental health conditions), with Motivational Interviewing the second most common (21\% of studies). The two most common mental health conditions the EBP was designed to treat was mood disorders (depression and/or anxiety; $39.2 \%$ ), followed by substance use disorders (21.4\%). Six eligible studies were rated as low risk of bias, 21 medium risk of bias, and one high risk of bias. Regarding implementation strategies included in EBP training methods, the most common strategies were making the training dynamic and providing clinical supervision, with only a few training methods including other strategies (e.g., relay of clinical data to providers, learning collaborative, etc.)

Appendix Table 2 includes an overview of the main findings reported in each study including effect and $p$ values sizes as available. Appendix Tables 3a-3c include data for each outcome extracted from each study. Differences (or lack thereof) between conditions summarized below are based on statistical significance. The synthesis of the included studies' results, described below, is organized by implementation outcomes [12] examined in the review. We categorized training conditions as (1) no training/self-study (participants in these conditions were randomized to waitlist, given a EBP treatment manual and/or didactic materials to study independently, or engaged in a placebo training, in which content unrelated to the EBP interventions was presented); (2) active training (participants were randomized to any type of active training such as in person workshop, online training, 
Table 2 Descriptive characteristics of included studies $(k=28)$

\begin{tabular}{|c|c|}
\hline Characteristic & $\begin{array}{l}n \text { and proportion (\%) } \\
\text { of included studies }\end{array}$ \\
\hline \multicolumn{2}{|l|}{ Provider sample size } \\
\hline$N<50$ & $9(32.1 \%)$ \\
\hline$N=50-100$ & $8(28.6 \%)$ \\
\hline$N>100$ & $11(39.2 \%)$ \\
\hline Provider sample included trainees/unlicensed individuals & $14(50.0 \%)$ \\
\hline Study included objective assessment of provider behavior & $16(57.1 \%)$ \\
\hline Study included knowledge test/self-report only & $11(39.2 \%)$ \\
\hline Study included patient outcomes only & $1(3.6 \%)$ \\
\hline \multicolumn{2}{|l|}{ EBPs } \\
\hline Cognitive behavioral therapy variant & $10(35.7 \%)$ \\
\hline Motivational interviewing & $6(21.4 \%)$ \\
\hline Exposure therapy & $4(14.3 \%)$ \\
\hline Dialectical behavior therapy & $3(10.7 \%)$ \\
\hline Behavioral activation & $2(7.1 \%)$ \\
\hline Contingency management & $1(3.6 \%)$ \\
\hline Cognitive processing therapy & $1(3.6 \%)$ \\
\hline Trauma-focused cognitive behavioral therapy & $1(3.6 \%)$ \\
\hline \multicolumn{2}{|l|}{ Mental health condition EBP designed for } \\
\hline Depression and/or anxiety disorders & $11(39.2 \%)$ \\
\hline Substance use disorders & $10(35.7 \%)$ \\
\hline Borderline personality disorder & $3(10.7 \%)$ \\
\hline Posttraumatic stress disorder & $3(10.7 \%)$ \\
\hline Eating disorders & $1(3.6 \%)$ \\
\hline
\end{tabular}

EBP evidence-based psychotherapy

Cognitive behavioral therapy variant refers to a range cognitive behavioral therapy protocols designed to treat different mental health conditions

distance learning, etc.). Active trainings could be either instructor-led, self-paced, or a combination of the two; or (3) active training plus consultation (participants were randomized to receive additional consultation after their initial training). A few studies in category 3 compared different consultation methods after participants received the same initial training. We describe the results for each outcome by comparisons of the following training conditions: active training compared to no training/ self-study, active training comparison, and active training compared to active training plus consultation.

\section{Implementation outcomes}

\section{Provider satisfaction with training}

Ten studies (36\%) evaluated provider satisfaction with training $[26,28,31-33,35-37,42,46]$.

\section{Active training compared to no training/self-study/} placebo Based on four studies [31-33, 36], we found moderate strength of evidence that more active training conditions resulted in greater satisfaction compared to self-guided study of treatment manual/training materials or placebo online training control condition. Specifically, one study found that both an in-person and online training had higher satisfaction ratings than self-study of the treatment manual [31]. Another found that the inperson training, but not the online training, was rated more satisfactory than self-study of the treatment manual [33]. The two other studies looked at two indices of satisfaction: acceptability (e.g., helpfulness of the training materials) and usability (e.g., ease of navigation through the training material). Both studies found that active online trainings were rated more acceptable than a placebo online training [32, 36]. Regarding usability, one study found no difference between the active online training conditions and the placebo online training condition [36]. The other study found that both the active and placebo online trainings were rated as more usable than the training manual [32].

Active training comparison Seven studies [26, 31, 33, $35,36,42,46]$ compared provider satisfaction with different active training methods. Overall strength of evidence was low for any active training condition 
Table 3 Discrete ERIC implementation strategies (Powell et al. 2015) used in EBP training methods in included studies

\begin{tabular}{|c|c|c|c|c|c|c|c|c|}
\hline \multirow{2}{*}{$\begin{array}{l}\text { Study (first } \\
\text { author and } \\
\text { year) }\end{array}$} & \multirow{2}{*}{$\begin{array}{l}\text { EBP } \\
\text { Mental health } \\
\text { condition }\end{array}$} & \multirow{2}{*}{$\begin{array}{l}\text { Training methods } \\
\text { evaluated }\end{array}$} & \multicolumn{6}{|c|}{ Implementation strategies } \\
\hline & & & $\begin{array}{l}\text { Distribute } \\
\text { educational } \\
\text { materials }\end{array}$ & $\begin{array}{l}\text { Facilitate } \\
\text { relay of } \\
\text { clinical } \\
\text { data to } \\
\text { providers }\end{array}$ & $\begin{array}{l}\text { Make } \\
\text { training } \\
\text { dynamic }\end{array}$ & $\begin{array}{l}\text { Provide } \\
\text { clinical } \\
\text { supervision }\end{array}$ & $\begin{array}{l}\text { Learning } \\
\text { collaborative }\end{array}$ & Other \\
\hline \multirow[t]{2}{*}{$\begin{array}{l}\text { Bearman } \\
2017[25]\end{array}$} & \multirow{2}{*}{$\begin{array}{l}\text { Cognitive behavioral } \\
\text { therapy } \\
\text { Youth depression }\end{array}$} & $\begin{array}{l}\text { In-person workshop + } \\
\text { experiential supervision }\end{array}$ & & & $x$ & $x$ & & $\begin{array}{l}\text { X (supervision } \\
\text { dynamic) }\end{array}$ \\
\hline & & $\begin{array}{l}\text { In-person workshop + } \\
\text { supervision as usual }\end{array}$ & & & $x$ & $x$ & & \\
\hline \multirow[t]{3}{*}{$\begin{array}{l}\text { Beidas } 2012 \\
{[26]}\end{array}$} & \multirow{3}{*}{$\begin{array}{l}\text { Cognitive behavioral } \\
\text { therapy, Coping Cat } \\
\text { protocol } \\
\text { Youth anxiety }\end{array}$} & $\begin{array}{l}\text { In person didactic } \\
\text { workshop + virtual } \\
\text { consultation }\end{array}$ & & & & $x$ & & \\
\hline & & $\begin{array}{l}\text { Online training + virtual } \\
\text { consultation }\end{array}$ & & & & $x$ & & \\
\hline & & $\begin{array}{l}\text { In-person didactic/experiential } \\
\text { workshop + virtual } \\
\text { consultation }\end{array}$ & & & $x$ & $x$ & & \\
\hline \multirow{2}{*}{$\begin{array}{l}\text { Bennett-Levy } \\
2012 \text { [27] }\end{array}$} & \multirow[b]{2}{*}{$\begin{array}{l}\text { Cognitive behavioral } \\
\text { therapy } \\
\text { Depression, panic } \\
\text { disorder, with } \\
\text { agoraphobia, } \\
\text { generalized anxiety } \\
\text { disorder }\end{array}$} & Online training & & & $x$ & & & \\
\hline & & $\begin{array}{l}\text { Online training + } \\
\text { supportive calls }\end{array}$ & & & $x$ & & & $\begin{array}{l}\text { X (supportive } \\
\text { calls to } \\
\text { providers) }\end{array}$ \\
\hline \multirow[t]{3}{*}{$\begin{array}{l}\text { Chu } 2017 \\
{[28]}\end{array}$} & \multirow[t]{3}{*}{$\begin{array}{l}\text { Cognitive behavioral } \\
\text { therapy } \\
\text { Youth anxiety }\end{array}$} & $\begin{array}{l}\text { Online training + } \\
\text { online expert streaming } \\
\text { consultation }\end{array}$ & & & & $\begin{array}{l}X \text { (watch } \\
\text { streamed) }\end{array}$ & & \\
\hline & & $\begin{array}{l}\text { Online training + in-person } \\
\text { peer consultation }\end{array}$ & & & & & $x$ & \\
\hline & & $\begin{array}{l}\text { Online training + fact sheet } \\
\text { self-study }\end{array}$ & $x$ & & & & & \\
\hline \multirow[t]{2}{*}{$\begin{array}{l}\text { Cohen } 2016 \\
{[29]}\end{array}$} & \multirow{2}{*}{$\begin{array}{l}\text { Trauma-focused } \\
\text { cognitive behavioral } \\
\text { therapy } \\
\text { PTSD and depression }\end{array}$} & $\begin{array}{l}\text { Online training }+ \text { online } \\
\text { consultation course }\end{array}$ & & & $x$ & $\begin{array}{l}\text { X } \\
\text { (automated) }\end{array}$ & & \\
\hline & & $\begin{array}{l}\text { Online training + in person } \\
\text { workshop + phone } \\
\text { consultation }\end{array}$ & & & $x$ & $x$ & & \\
\hline \multirow{2}{*}{$\begin{array}{l}\text { Cooper } 2017 \\
{[30]}\end{array}$} & \multirow{2}{*}{$\begin{array}{l}\text { Cognitive behavioral } \\
\text { therapy } \\
\text { Eating disorders }\end{array}$} & Online training & & & $x$ & & & \\
\hline & & $\begin{array}{l}\text { Online training + supportive } \\
\text { calls }\end{array}$ & & & $x$ & & & $\begin{array}{l}\text { X (supportive } \\
\text { calls to } \\
\text { providers) }\end{array}$ \\
\hline \multirow{3}{*}{$\begin{array}{l}\text { Dimeff } 2009 \\
{[31]}\end{array}$} & \multirow{3}{*}{$\begin{array}{l}\text { Dialectical behavior } \\
\text { therapy } \\
\text { Borderline personality } \\
\text { disorder }\end{array}$} & Online training & & & $x$ & & & \\
\hline & & In-person workshop & & & & & & \\
\hline & & Treatment manual & $x$ & & & & & \\
\hline \multirow{3}{*}{$\begin{array}{l}\text { Dimeff } 2011 \\
{[32]}\end{array}$} & \multirow{3}{*}{$\begin{array}{l}\text { Dialectical behavior } \\
\text { therapy } \\
\text { Borderline personality } \\
\text { disorder }\end{array}$} & Online training & & & $x$ & & & \\
\hline & & Placebo online training & & & & & & \\
\hline & & Treatment manual & $x$ & & & & & \\
\hline \multirow{3}{*}{$\begin{array}{l}\text { Dimeff } 2015 \\
\text { [33] }\end{array}$} & \multirow{3}{*}{$\begin{array}{l}\text { Dialectical behavior } \\
\text { therapy } \\
\text { Borderline personality } \\
\text { disorder }\end{array}$} & Online training & & & $x$ & & & \\
\hline & & In-person workshop & & & $x$ & & & \\
\hline & & Treatment manual & $x$ & & & & & \\
\hline \multirow[t]{2}{*}{ Fu 2015 [34] } & \multirow{2}{*}{$\begin{array}{l}\text { Motivational } \\
\text { interviewing } \\
\text { Smoking cessation }\end{array}$} & In-person workshop & & & $x$ & & & \\
\hline & & $\begin{array}{l}\text { In-person workshop + } \\
\text { booster sessions }+ \\
\text { peer coaching }\end{array}$ & & & $x$ & $x$ & $x$ & \\
\hline Gega 2007 & Exposure therapy & Online training & & & & & & \\
\hline
\end{tabular}


Table 3 Discrete ERIC implementation strategies (Powell et al. 2015) used in EBP training methods in included studies (Continued)

\begin{tabular}{|c|c|c|c|c|c|c|c|c|}
\hline \multirow{2}{*}{$\begin{array}{l}\text { Study (first } \\
\text { author and } \\
\text { year) }\end{array}$} & \multirow{2}{*}{$\begin{array}{l}\text { EBP } \\
\text { Mental health } \\
\text { condition }\end{array}$} & \multirow{2}{*}{$\begin{array}{l}\text { Training methods } \\
\text { evaluated }\end{array}$} & \multicolumn{6}{|c|}{ Implementation strategies } \\
\hline & & & $\begin{array}{l}\text { Distribute } \\
\text { educational } \\
\text { materials }\end{array}$ & $\begin{array}{l}\text { Facilitate } \\
\text { relay of } \\
\text { clinical } \\
\text { data to } \\
\text { providers }\end{array}$ & $\begin{array}{l}\text { Make } \\
\text { training } \\
\text { dynamic }\end{array}$ & $\begin{array}{l}\text { Provide } \\
\text { clinical } \\
\text { supervision }\end{array}$ & $\begin{array}{l}\text { Learning } \\
\text { collaborative }\end{array}$ & Other \\
\hline [35] & Phobias & In-person workshop & & & & & & \\
\hline \multirow{3}{*}{$\begin{array}{l}\text { Harned } 2011 \\
{[36]}\end{array}$} & \multirow{3}{*}{$\begin{array}{l}\text { Exposure therapy } \\
\text { Anxiety disorders }\end{array}$} & Online training & & & $x$ & & & \\
\hline & & $\begin{array}{l}\text { Online training + } \\
\text { motivational } \\
\text { interviewing }\end{array}$ & & & $x$ & & & $\begin{array}{l}X \text { (motivational } \\
\text { component } \\
\text { for providers } \\
\text { to adopt EBP) }\end{array}$ \\
\hline & & $\begin{array}{l}\text { Placebo online } \\
\text { training }\end{array}$ & & & & & & \\
\hline \multirow{3}{*}{$\begin{array}{l}\text { Harned } 2014 \\
\text { [37] }\end{array}$} & \multirow{3}{*}{$\begin{array}{l}\text { Exposure therapy } \\
\text { Anxiety disorders }\end{array}$} & Online training & & & $x$ & & & \\
\hline & & $\begin{array}{l}\text { Online training + } \\
\text { motivational } \\
\text { enhancement }\end{array}$ & & & $x$ & & & $\begin{array}{l}\text { X (motivational } \\
\text { component } \\
\text { for providers } \\
\text { to adopt EBP) }\end{array}$ \\
\hline & & $\begin{array}{l}\text { Online training + } \\
\text { motivational } \\
\text { enhancement + learning } \\
\text { community }\end{array}$ & & & & & $\begin{array}{l}X \text { (with } \\
\text { expert) }\end{array}$ & \\
\hline \multirow{2}{*}{$\begin{array}{l}\text { Henggeler } \\
2008[38]\end{array}$} & \multirow{2}{*}{$\begin{array}{l}\text { Contingency } \\
\text { management } \\
\text { Marijuana abuse }\end{array}$} & In-person workshop & & & $x$ & & & \\
\hline & & $\begin{array}{l}\text { In-person workshop + } \\
\text { Intensive Quality Assurance }\end{array}$ & & & $x$ & $x$ & & \\
\hline \multirow{2}{*}{$\begin{array}{l}\text { Hubley } 2015 \\
\text { [39] }\end{array}$} & \multirow{2}{*}{$\begin{array}{l}\text { Behavioral activation } \\
\text { Depression }\end{array}$} & Online training & & & & & & \\
\hline & & $\begin{array}{l}\text { Online placebo control } \\
\text { training }\end{array}$ & & & & & & \\
\hline \multirow[t]{2}{*}{$\begin{array}{l}\text { Larson } 2013 \\
{[40]}\end{array}$} & \multirow{2}{*}{$\begin{array}{l}\text { Cognitive behavioral } \\
\text { therapy } \\
\text { Substance abuse }\end{array}$} & $\begin{array}{l}\text { Online training + } \\
\text { supervision }\end{array}$ & & & $x$ & $x$ & & \\
\hline & & $\begin{array}{l}\text { Training manual + } \\
\text { supervision }\end{array}$ & $x$ & & & $x$ & & \\
\hline \multirow[t]{3}{*}{$\begin{array}{l}\text { Martino } 2011 \\
\text { [41] }\end{array}$} & \multirow[t]{3}{*}{$\begin{array}{l}\text { Motivational } \\
\text { interviewing } \\
\text { Substance abuse }\end{array}$} & $\begin{array}{l}\text { In person workshop } \\
\text { by expert }+ \\
\text { consultation }\end{array}$ & & $x$ & & $x$ & & \\
\hline & & $\begin{array}{l}\text { In person workshop by trainee } \\
+ \text { consultation }\end{array}$ & & $x$ & & $x$ & & $\begin{array}{l}X \text { (train-the- } \\
\text { trainer) }\end{array}$ \\
\hline & & $\begin{array}{l}\text { Treatment manual + didactic } \\
\text { materials }\end{array}$ & $x$ & & & & & \\
\hline \multirow[t]{2}{*}{$\begin{array}{l}\text { McDonough } \\
2002[42]\end{array}$} & \multirow[t]{2}{*}{$\begin{array}{l}\text { Exposure therapy } \\
\text { Phobias }\end{array}$} & $\begin{array}{l}\text { In-person lecture + } \\
\text { online training }\end{array}$ & & & $x$ & & & \\
\hline & & $\begin{array}{l}\text { In-person lecture }+ \\
\text { in-person group tutorial }\end{array}$ & & & $x$ & & & \\
\hline \multirow{5}{*}{$\begin{array}{l}\text { Miller } 2004 \\
{[43]}\end{array}$} & \multirow{5}{*}{$\begin{array}{l}\text { Motivational } \\
\text { interviewing } \\
\text { Substance abuse }\end{array}$} & In-person workshop & & & $x$ & & & \\
\hline & & $\begin{array}{l}\text { In-person workshop + audio } \\
\text { review and feedback }\end{array}$ & & $x$ & $x$ & & & \\
\hline & & $\begin{array}{l}\text { In-person workshop + } \\
\text { coaching }\end{array}$ & & & $x$ & $x$ & & \\
\hline & & $\begin{array}{l}\text { In-person workshop + audio } \\
\text { review and feedback + } \\
\text { coaching }\end{array}$ & & $x$ & $x$ & $x$ & & \\
\hline & & $\begin{array}{l}\text { Training manual + didactic } \\
\text { videotapes }\end{array}$ & $x$ & & $x$ & & & \\
\hline $\begin{array}{l}\text { Monson } \\
2018[44]\end{array}$ & $\begin{array}{l}\text { Cognitive processing } \\
\text { therapy }\end{array}$ & In-person workshop & & & & & & \\
\hline
\end{tabular}


Table 3 Discrete ERIC implementation strategies (Powell et al. 2015) used in EBP training methods in included studies (Continued)

\begin{tabular}{|c|c|c|c|c|c|c|c|c|}
\hline \multirow{2}{*}{$\begin{array}{l}\text { Study (first } \\
\text { author and } \\
\text { year) }\end{array}$} & \multirow{2}{*}{$\begin{array}{l}\text { EBP } \\
\text { Mental health } \\
\text { condition }\end{array}$} & \multirow{2}{*}{$\begin{array}{l}\text { Training methods } \\
\text { evaluated }\end{array}$} & \multicolumn{6}{|c|}{ Implementation strategies } \\
\hline & & & $\begin{array}{l}\text { Distribute } \\
\text { educational } \\
\text { materials }\end{array}$ & $\begin{array}{l}\text { Facilitate } \\
\text { relay of } \\
\text { clinical } \\
\text { data to } \\
\text { providers }\end{array}$ & $\begin{array}{l}\text { Make } \\
\text { training } \\
\text { dynamic }\end{array}$ & $\begin{array}{l}\text { Provide } \\
\text { clinical } \\
\text { supervision }\end{array}$ & $\begin{array}{l}\text { Learning } \\
\text { collaborative }\end{array}$ & Other \\
\hline & $\begin{array}{l}\text { Posttraumatic } \\
\text { stress disorder }\end{array}$ & $\begin{array}{l}\text { In-person workshop + } \\
\text { consultation }\end{array}$ & & & & $x$ & & \\
\hline & & $\begin{array}{l}\text { In-person workshop }+ \\
\text { consultation with session } \\
\text { audio review }\end{array}$ & & $x$ & & $x$ & & $\begin{array}{l}\text { X (relay of } \\
\text { clinical data } \\
\text { in supervision) }\end{array}$ \\
\hline \multirow{3}{*}{$\begin{array}{l}\text { Moyers } 2008 \\
{[45]}\end{array}$} & \multirow{3}{*}{$\begin{array}{l}\text { Motivational } \\
\text { interviewing } \\
\text { Substance } \\
\text { abuse }\end{array}$} & In-person workshop & & & & & & \\
\hline & & $\begin{array}{l}\text { In-person workshop + } \\
\text { feedback }+ \text { consultation }\end{array}$ & & $x$ & & $x$ & & \\
\hline & & $\begin{array}{l}\text { Training manual + didactic } \\
\text { videotapes }\end{array}$ & $x$ & & & & & \\
\hline \multirow{2}{*}{$\begin{array}{l}\text { Puspitasari } \\
2017 \text { [46] }\end{array}$} & \multirow{2}{*}{$\begin{array}{l}\text { Behavioral } \\
\text { activation } \\
\text { Depression }\end{array}$} & Distance workshop & & & $x$ & & & \\
\hline & & Online training & & & & & & \\
\hline \multirow[t]{3}{*}{$\begin{array}{l}\text { Rakovshik } \\
2016 \text { [47] }\end{array}$} & \multirow{3}{*}{$\begin{array}{l}\text { Cognitive } \\
\text { behavioral therapy } \\
\text { Panic disorder w/ } \\
\text { agoraphobia, } \\
\text { obsessive- } \\
\text { compulsive } \\
\text { disorder }\end{array}$} & $\begin{array}{l}\text { Online training }+ \\
\text { consultation worksheet }\end{array}$ & $x$ & & $x$ & & & \\
\hline & & $\begin{array}{l}\text { Online training }+ \\
\text { consultation worksheet + } \\
\text { remote supervision }\end{array}$ & & & $x$ & $x$ & & \\
\hline & & Delayed training control & & & & & & \\
\hline \multirow[t]{3}{*}{$\begin{array}{l}\text { Rawson } 2013 \\
{[48]}\end{array}$} & \multirow{3}{*}{$\begin{array}{l}\text { Cognitive behavioral } \\
\text { therapy } \\
\text { Stimulant } \\
\text { dependence }\end{array}$} & $\begin{array}{l}\text { In-person workshop + } \\
\text { in-person coaching }\end{array}$ & & & $x$ & $x$ & & \\
\hline & & $\begin{array}{l}\text { Distance workshop + } \\
\text { telephone coaching }\end{array}$ & & & $x$ & $x$ & & \\
\hline & & $\begin{array}{l}\text { Training manual + } \\
\text { orientation }\end{array}$ & $x$ & & & & & \\
\hline \multirow{3}{*}{$\begin{array}{l}\text { Ruzek } 2014 \\
\text { [49] }\end{array}$} & \multirow{3}{*}{$\begin{array}{l}\text { Cognitive behavioral } \\
\text { therapy skills } \\
\text { Posttraumatic stress } \\
\text { disorder }\end{array}$} & Online training & & & $x$ & & & \\
\hline & & $\begin{array}{l}\text { Online training }+ \\
\text { telephone consultation }\end{array}$ & & & $x$ & $x$ & & \\
\hline & & No training control & & & & & & \\
\hline \multirow{3}{*}{$\begin{array}{l}\text { Smith } 2012 \\
\text { [50] }\end{array}$} & \multirow{3}{*}{$\begin{array}{l}\text { Motivational } \\
\text { Interviewing } \\
\text { Substance abuse }\end{array}$} & In-person workshop & & & $x$ & & & \\
\hline & & $\begin{array}{l}\text { In-person workshop + } \\
\text { session audio review }\end{array}$ & & $x$ & $x$ & & & \\
\hline & & $\begin{array}{l}\text { In-person workshop + } \\
\text { live teleconferencing } \\
\text { supervision }\end{array}$ & & & $x$ & $x$ & & \\
\hline \multirow{3}{*}{$\begin{array}{l}\text { Weingardt } \\
2006[51]\end{array}$} & \multirow{3}{*}{$\begin{array}{l}\text { Cognitive behavioral } \\
\text { therapy } \\
\text { Cocaine addiction }\end{array}$} & In-person workshop & & & & & & \\
\hline & & Online training & & & & & & \\
\hline & & $\begin{array}{l}\text { Delayed training } \\
\text { control }\end{array}$ & & & & & & \\
\hline \multirow[t]{2}{*}{$\begin{array}{l}\text { Zatzick } 2014 \\
\text { [52] }\end{array}$} & \multirow{2}{*}{$\begin{array}{l}\text { Motivational } \\
\text { Interviewing } \\
\text { Problem drinking }\end{array}$} & $\begin{array}{l}\text { In-person workshop + } \\
\text { feedback + coaching }\end{array}$ & & $x$ & & $x$ & & \\
\hline & & No training & & & & & & \\
\hline
\end{tabular}

resulting in greater satisfaction than another. Four studies [31, 35, 36, 46], two of which compared online and in-person training, one which compared online training to an enhanced online version, and one which compared distance workshop (remote training with live instructor) to online training, found no differences in satisfaction between active training methods. Three studies [26, 33, 42] found that participants were more satisfied with in-person training compared to online training. 
Active training compared to active training plus consultation Two studies [28, 37] examined provider satisfaction regarding consultation after training. Results were mixed and strength of evidence rated as insufficient. One study found no difference in satisfaction between two active consultation conditions and a noconsultation control [28]. Another study found that the motivational enhancement element of the training intervention, aimed at addressing providers' potential attitudinal barriers to learning and using the EBP, was rated as more acceptable when it was combined with an online learning collaborative [37].

\section{Provider EBP treatment knowledge}

Sixteen studies (57\%) evaluated provider EBP treatment knowledge [26-28, 30-37, 39, 42, 48, 49, 51].

Active training compared to no training/self-study/ placebo Eight studies compared change in treatment knowledge between one or more active training conditions and a no-training, self-guided study of treatment manual/training materials, or placebo online training control condition [31-33, 36, 39, 48, 49, 51]. All studies found at least one active training condition resulted in greater gains in treatment knowledge than the control condition (moderate strength of evidence).

Active training comparison Eleven studies compared gains in treatment knowledge between different active training methods $[26,27,30,31,33,35-37,42,48,51]$. Strength of evidence was rated low for any active training method resulting in increased treatment knowledge over another active method. Nine found no differences in treatment knowledge between active training conditions $[26,35-37,42,48,51]$, including five studies that compared online and in-person training [26, 35, 42, 48, 51], two studies that compared online training to an enhanced online version [36, 37], and two studies that compared online training to online training with supportive phone calls $[27,30]$. Dimeff $(2009,2015)$ did find a difference between active training conditions: specifically, online training conditions resulted in greater increases in treatment knowledge than expert-led, in-person training $[31,33]$.

Active training compared to active training plus consultation Four studies compared the additive effects of consultation on treatment knowledge beyond initial training or compared different consultation methods and overall strength of evidence was low [28, 34, 37, 49]. One study found that treatment knowledge was similar between participants receiving different types of consultation (online expert-led consultation, in-person peer consultation, or self-study of fact sheet control); notably, participants in all three conditions had decreases in treatment knowledge pre- to post-consultation/selfstudy of fact sheet [28]. Three studies found that adding expert consultation (two after online training and one after in-person training) led to greater increases in treatment knowledge in at least some domains compared to completing the initial training only $[34,37,49]$.

\section{Provider skill acquisition/adherence}

Fifteen studies (54\%) evaluated provider skill acquisition/EBP adherence [26-29, 31, 34, 38, 40, 41, 43, 45, 48-50, 52].

Active training compared to no training/self-study/ placebo Six studies compared one or more active training conditions to a no-training or self-guided study of treatment manual/training materials control condition $[41,43,45,48,49,52]$. We found a moderate strength of evidence that more active training resulted in improved adherence. Five out of six studies found at least one active training condition resulted in greater gains in EBP adherence than the control condition [41, 43, 48, 49, 52]; one study found no difference in skill acquisition between participants who attended an in-person workshop and those who engaged in self-study of training materials [45].

Active training comparison Five studies compared gains in skill acquisition/adherence between different active training methods and all found no difference in adherence between active training conditions [26, 27, 40,41, 48] (low strength of evidence).

Active training compared to active training plus consultation Seven studies compared the additive effects of consultation beyond initial training or compared different consultation methods on skill acquisition/adherence [29, 34, 38, 43, 45, 49, 50]. We found low strength of evidence that active training with consultation resulted in greater EBP adherence than active training alone. Two studies found that adding consultation (up to six, 30-min consultation calls) did not lead to increases in EBP adherence compared to an in-person workshop alone $[43,45]$. Five studies found that at least one form of consultation did lead to greater gains than training alone (consultation dosage in these studies ranged from five sessions over 5 weeks to weekly sessions over 6 months) $[29,34,38,49,50]$. Of note, one of these studies used provider self-report [29] and one used client report of provider behavior [38] to measure adherence, which may not be as valid as objective coding of provider behavior. Additionally, Ruzek (2014) found that online training plus consultation (up to six, 45- to 60min consultation calls) led to increased adherence in only one out of three of the EBP skills taught compared to online training only [49]. 


\section{Provider competence}

Fourteen studies $(50 \%)$ evaluated change in provider competence [25, 26, 31, 33, 34, 37, 41, 43, 45-48, 50, 52].

Active training compared to no training/self-study/ placebo Eight studies [31, 33, 41, 43, 45, 47, 48, 52] compared one or more active training conditions to a no-training or self-guided study of treatment manual/ training materials control condition. Overall strength of evidence was rated low. Of those eight, five found that at least one active training condition led to greater increases in provider competence than no training/self-guided study $[41,43,45,48,52]$. Specifically, all five studies found that an expert-led in-person workshop was superior to selfguided study in increasing provider competence. Three studies, however, found no difference between at least one active training condition (online training or expert-led inperson training) and no-training/self-guided study control condition [31, 33, 47].

Active training comparison Seven studies compared the effects of different active training methods on provider competence and strength of evidence for the superiority of one active training method improving competence over another was low $[26,31,33,37,41,46,48]$. Five of the seven studies found no differences in change in provider competence between training methods (three studies compared in-person to online training, one study compared online training to an enhanced online training, and one study compared in-person to distance learning). Participants in all conditions had an increase in competence pre- to post-training/follow-up [26, 31, 33, 37, 48]. Two studies found differences between active training conditions: Puspitasari (2017) found that an expert-led, live distance (online) training led to greater gains in Behavioral Activation skills competence compared to a self-paced online training [46]. Martino (2011) found that an expert-led in-person training and consultation, compared to "trainthe-trainer"-led in-person training and consultation, led to greater increases in fundamental competence of Motivational Interviewing in role-played sessions, but not client sessions, from pre-training to 12-week follow-up [41].

Active training compared to active training plus consultation Eight studies compared the additive effects of consultation beyond initial training or compared different consultation methods $[25,34,37,43,45,47,48,50]$. Overall strength of evidence was rated as low. Five studies compared a training plus consultation condition to a no consultation (initial training only) condition [34, 37, 43, 45, 47]. Three of these studies found that adding consultation (ranging from three to eight consultation sessions) in addition to training (one study post in-person training, two post online training) resulted in greater increases in provider competence compared to training only [34, 37, 47]. However, two studies found that consultation did not result in additional benefit regarding provider competence beyond the initial in-person training [43, 45], although in Miller (2004), only the conditions with consultation had average competency scores at the clinical proficiency standard.

Three studies compared the effect of different active consultation conditions on provider competence. One study found no differences between consultation conditions (practice feedback, individual consultation sessions, or both) [43]. Two studies found differences: Bearman (2017) found an experiential, active consultation led to greater increases in provider competence and EBP expertise compared to traditional consultation [25], and Rawson (2013) found that in-person consultation led to greater increases in provider competence than telephone consultation and a higher level of competence at a 24-week follow-up [48].

\section{Provider fidelity}

Only one study evaluated change in providers' EBP fidelity (a composite adherence and competence rating) so strength of evidence was rated as insufficient. Bearman 2017 found enhanced supervision after an in-person workshop, which utilized experiential learning (e.g., modeling and role-plays) and provided feedback on session recordings, resulted in greater increases in provider EBP fidelity compared to supervision as usual post in-person workshop [25].

\section{EBP adoption}

Nine studies (32\%) evaluated adoption of the EBP in clinical practice. All of the studies measured adoption via provider self-reported use of EBP techniques through teaching and/or with clients [27, 28, 31-33, 36, 37, 46, 49].

Active training compared to no training/self-study/ placebo Based on five studies comparing adoption of EBP into clinical practice between one or more active training conditions and a no-training, self-guided study of treatment manual/training materials, or placebo online training control condition [31-33, 36, 49], active training may not have increased EBP adoption (low strength of evidence). Four of the five studies found that the active training condition did not result in greater adoption of EBP components than control (assessment of adoption ranged from immediately post-training to 90-days post-training) [31, 33, 36, 49]. Dimeff (2011) found that online training led to greater self-reported teaching and/or use of EBP skills learned compared to online placebo training at all four follow-up timepoints (2-weeks to 15-weeks post-training); however, it only led to greater teaching/use of EBP skills at one timepoint compared to self-study of the treatment manual [32]. 
Active training comparison Five studies compared EBP adoption between different active training methods [27, 31, 33, 37, 46]. None of the five studies found a difference in adoption between the active training conditions (three compared online to inperson training, two compared online training to an enhanced or supported online training) (low strength of evidence).

Active training compared to active training plus consultation Three studies compared the additive effects of consultation beyond initial training or different consultation methods on EBP adoption [28, 37, 49]. None found a difference between conditions in regard to EBP adoption (low strength of evidence).

\section{Costs}

Three studies (11\%) reported on EBP training costs. Rawson (2013) presented costs for provider training in an EBP (Cognitive Behavioral Therapy for stimulant dependence) for the three training conditions examined. The highest cost training method was in-person workshop plus in-person consultation (\$1485 per participant), followed by distance workshop plus telephone consultation (\$768 per participant), followed by providing participants with the training manual and an orientation session (\$145 per participant) [48]. Two other studies presented the costs associated with the active training condition studied (online training in Cognitive Behavioral Therapy: $\$ 440$ per participant [27]; in-person training plus feedback and consultation in Motivational Interviewing: $\$ 4300$ per site [52]). No studies examined the cost of training as it related to the comparative effectiveness of the training methods studied.

\section{Client outcomes}

Three studies (11\%) reported on client clinical outcomes in relation to EBP training $[29,44,52]$ and strength of evidence was rated as insufficient. One study found that clients in sites where providers were trained in the EBP (Motivational Interviewing) had a greater reduction in hazardous drinking scores in the year after hospitalization than clients in sites where providers were not trained [52]. Another study found that, after controlling for provider effects, clients were more likely to complete treatment (Trauma-focused Cognitive Behavioral Therapy) from providers who were randomized to complete online training and receive consultation compared to providers who were randomized to complete online training only [29]. The third study found that an expert-led in-person workshop in an EBP (Cognitive Processing Therapy) plus standard consultation resulted in greater reductions in client PTSD symptoms compared to an in-person workshop only. However, no difference was found in client outcomes between inperson workshop only and in-person workshop plus consultation with audio review [44].

\section{Discussion}

Active EBP training probably leads to greater increases in provider EBP knowledge and adherence and providers are probably more satisfied with training compared to no training, placebo training, or self-study of treatment materials. However, it is unclear if these provider-level implementation outcomes (e.g., satisfaction, treatment knowledge) translate to more effective delivery of EBPs and better client outcomes.

Findings were mixed regarding whether EBP training led to greater increases in competence, and no difference was found in EBP adoption between providers who received EBP training and those who did not. Thus, it may be that although training in EBPs leads to increases in providers' ability to employ foundational elements of EBP delivery (e.g., treatment knowledge and skill acquisition/adherence), it does not result in higher quality delivery of the treatment (e.g., competence). Competent, skillful delivery of the treatment may be more difficult to teach in a relatively brief EBP training context, and training in these more nuanced therapeutic skills may need to begin earlier in providers' education or be further developed through additional practice, consultation, or other supports. Additionally, no specific EBP training modality (e.g., in-person training, online training) was more effective than another with regard to increasing provider EBP knowledge, skill acquisition/adherence, competence, adoption, or satisfaction.

In contrast to the general conclusions of previous reviews $[15,16]$, the additional benefit of consultation beyond initial EBP training was inconclusive. Specifically, no difference was found in EBP adoption between those who received active training and those who received active training plus consultation, and findings were mixed regarding the additive benefit of consultation on provider EBP knowledge, adherence/skill acquisition, competence, and satisfaction. Our findings may differ from the previous reviews $[15,16]$ in that, in addition to including newer research, only studies using randomized controlled designs were included which, taken together, provide inconclusive support for the additional contribution of consultation. Differences in consultation "dose" and intensity may have contributed to these mixed findings. Individual studies point to elements of consultation that may be particularly effective, such as performance feedback, modeling, and role-playing [25], and others that may detract, such as audio review in a group consultation setting [44]. However, more research is needed to identify effective consultation practices that lead to 
providers' continued skill development over time. Moreover, the finding that providers trained in EBPs and provided with additional consultation may not be more likely to adopt EBPs than those who are not trained or only engage in self-study is concerning. Underutilization of EBPs is problematic considering the extensive resources employed to train providers in EBPs in an effort to increase access to effective treatment. Prior work with other types of clinical innovations have consistently demonstrated that educational workshops alone is insufficient to impact complex behavior change [53] and implementation strategies beyond provider training are needed for successful implementation. Making the training dynamic and post-training clinical supervision/ consultation were the most widely used strategies and most studies reported using few other implementation strategies [14]. These findings add to that literature by clarifying that specific implementation strategies beyond those focused on enhancing clinical knowledge and skill may be needed. Current initiatives, such as the VHA's PTSD Consultation Program, which provides ongoing, free continuing education and consultation to ensure high-quality EBP delivery for PTSD, is a recent example of an EBP training initiative broadening clinical training to encompass more implementation strategies; evaluation of implementation outcomes of such programs is warranted [54]. To ensure continued utilization of EBPs after training and consultation have ended, more focus is needed on modifying providers' negative beliefs about EBPs and utilizing novel behavioral strategies to increase provider adoption of EBPs [55, 56]. Additionally, further study is necessary to understand the larger contextual factors (e.g., health care systems, organizational, and team factors) that may influence the use of EBPs [57].

While this review captured a broad range of studies evaluating different EBP training methods, there was substantial heterogeneity among studies, making it difficult to compare results across studies or draw conclusions about the effectiveness of specific training methods. Studies included in the review evaluated training methods for different EBPs, included providers at different training levels and with different levels of exposure to the EBP, and evaluated a diverse set of training and consultation methods. Future research should examine the effect of provider background and training, EBP type, and client mental health conditions, on EBP training outcomes.

Furthermore, the definitions and measurement of the provider-centered outcomes differed across studies (see Appendix Table 1 for a detailed description of these elements for each study). While the studies included in this review all utilized a strong research design (i.e., randomized controlled design), and with one exception [47], were rated as having a low to medium risk of bias, the strength of evidence for outcomes of interest was often limited due a lack of consistency in the direction of effects (e.g., some studies finding statistically significant differences between training conditions and others having null results). Additionally, strength of evidence was rated as insufficient for some outcomes due to the low number of studies (e.g., one or two) evaluating the outcome, or because studies defined outcomes differently, and thus, had limited convergent evidence. Thus, a lack of consistency in direction of effects across studies may be accounted for, in part, by variability in construct definitions and measurement rather than by effectiveness of training method. Future research in this area should focus on the development and use of uniform measures of EBP treatment adherence and competence whenever possible. Finally, the follow-up assessment timeframes of many of the studies included in the review were relatively short, potentially skewing findings about provider gains. Longer-term follow-up would allow for assessment of durability of provider gains over time. Importantly, recent research has demonstrated that providers' EBP adherence is not static and often varies between sessions [58]; thus, assessment of provider adherence and competence may be best evaluated using multiple data points over the course of treatment. Given the limitations of the current body of evidence, conclusions about the most effective EBP training methods should be drawn with caution.

Notably, the majority of studies included in the review did not measure several important outcomes. First, there is an overall dearth of research evaluating the effect of training on client clinical outcomes, the outcome of greatest importance. Secondly, no studies evaluated changes in implementation or client outcomes from different training methods with regards to costs, such as instructor and provider time spent in initial training activities and post-training consultation. Extrapolating from costs reported across studies, inperson workshops followed by in-person consultation may be over three times more expensive than online training and over ten times more expensive than providing treatment manuals [27, 48]. Future studies should report costs for each training method to allow for analysis of the impact of increasingly more resource-intensive training methods (e.g., expert-led inperson training with ongoing consultation) on provider and client outcomes achieved with higher levels of resource investment. This would allow stakeholders, especially those in low-resourced organizations (e.g., community mental health clinic directors), to determine which training initiatives are worth the investment.

It is important to note the limitations of the review itself. Limitations of the review include the use of two 
databases and a non-exhaustive list of training terms or psychotherapy approaches (e.g., did not include family therapies or parenting interventions), which may have resulted in the omission of relevant studies.

\section{Conclusions}

In summary, there is evidence that EBP training leads to short-term gains in the provider outcomes of EBP knowledge acquisition, adherence, and satisfaction compared to no training, placebo training, or self-study of treatment materials. Results were mixed for the effect of EBP training on provider competence and no effect was found for EBP adoption. No EBP training method demonstrated clear superiority over others. Additionally, the additive benefit and essential elements of post-training consultation are unclear. Given the absence of strong evidence regarding the most effective training methods, health systems should consider other factors when selecting an EBP training method, such as organizational costs (e.g., financial resources, provider productivity). Additionally, both providers and organization stakeholders should be aware that participation in an EBP training may not be sufficient to ensure delivery of highquality EBPs to clients.

\section{Supplementary information}

Supplementary information accompanies this paper at https://doi.org/10. 1186/s13012-020-00998-w.

Additional file 1: Appendix: Table 1. Study descriptions and Cochrane risk of bias rating. Table $\mathbf{2}$ Overview of included studies' findings $(k=28)$. Table 3a Study outcomes: participant adherence/EBP skill acquisition, competence, and fidelity. Table $\mathbf{3 b}$ Study outcomes: participant satisfaction, EBP treatment knowledge, and EBP adoption. Table 3c Study outcomes: client clinical outcomes and costs of training.

\section{Abbreviations}

EBP: Evidence-based psychotherapy; NCTSN: National Child Traumatic Stress Network; PRISMA: Preferred Reporting Items for Systematic Reviews and Meta-Analyses; PROSPERO: International Prospective Register of Systematic Reviews; SAMHSA: Substance Abuse and Mental Health Services Administration; VA: Department of Veterans Affairs; VHA: Veterans Health Administration

\section{Acknowledgements}

Not applicable.

\section{Authors' contributions}

HVM conceived of the systematic review and engaged in all aspects of study design and execution and drafted the manuscript. NG provided guidance on systematic review methodology and study design, assisted with data extraction, and gave feedback on manuscript drafts. LM provided feedback on study design, collaborated on search strategy, assisted with data extraction and risk of bias/strength of evidence ratings, helped with manuscript preparation, and provided feedback on manuscript drafts. LH provided feedback on study design, assisted with data extraction, helped with manuscript preparation, and gave feedback on manuscript drafts. TQS provided feedback on study design, assisted with data extraction, and gave feedback on manuscript drafts. SWS provided quidance on study design and feedback on manuscript drafts. TJW provided guidance on systematic review methodology and study design and gave feedback on manuscript drafts. SMKF provided guidance on systematic review methodology and study design, assisted with study selection and data extraction, and gave feedback on manuscript drafts. All authors read and approved the final manuscript.

\section{Authors' information}

This work supported with resources from the Center for Care Delivery and Outcomes Research at the Minneapolis VA Healthcare System. The views expressed in this article are those of the authors and do not reflect the Department of Veterans Affairs or the United States government.

\section{Funding}

This manuscript is based upon work supported by the Minneapolis VA Center for Care Delivery and Outcomes Research Locally Initiated Project Award. The funding body was not involved in the design of the study, collection, analysis, and interpretation of data, or in writing the manuscript.

Availability of data and materials

All articles included in this systematic review are publicly available.

Ethics approval and consent to participate

Not applicable.

Consent for publication

Not applicable.

\section{Competing interests}

The authors declare that they have no competing interests.

\section{Author details}

'Department of Psychiatry and Behavioral Sciences, University of Minnesota Medical School, 2450 Riverside Avenue, F282/2A West, Minneapolis, MN 55454, USA. ${ }^{2}$ Center for Care Delivery and Outcomes Research, Minneapolis VA Health Care System, Minneapolis, MN, USA. ${ }^{3}$ Minneapolis VA Health Care System, Minneapolis, MN, USA. ${ }^{4}$ University of St. Thomas, Minneapolis, MN, USA. ${ }^{5}$ Oscar G. Johnson VA Medical Center, Iron Mountain, MI, USA. ${ }^{6}$ National Center for PTSD Dissemination and Training Division, VA Palo Alto Healthcare System, Palo Alto, CA, USA. 7 Stanford University, Palo Alto, CA, USA. ${ }^{8}$ Department of Medicine, University of Minnesota School of Medicine, Minneapolis, MN, USA. ${ }^{9}$ National Center for PTSD Women's Health Sciences Division, VA Boston Healthcare System, Boston, MA, USA.

Received: 29 October 2019 Accepted: 7 May 2020

Published online: 27 May 2020

\section{References}

1. Chambless DL, Ollendick TH. Empirically supported psychological interventions: controversies and evidence. Annu Rev Psychol. 2001;52(1): 685-716.

2. McHugh RK, Barlow DH. The dissemination and implementation of evidence-based psychological treatments: a review of current efforts. Am Psychol. 2010;65(2):73-84.

3. Karlin BE, Ruzek JI, Chard KM, Eftekhari A, Monson CM, Hembree EA, et al. Dissemination of evidence-based psychological treatments for posttraumatic stress disorder in the veterans health administration. J Trauma Stress. 2010 Dec;23(6):663-73.

4. Trupin $\mathrm{E}$, Kerns $\mathrm{S}$. Introduction to the special issue: legislation related to children's evidence-based practice. Adm Policy Ment Health Ment Health Serv Res. 2017:44(1):1-5.

5. Beidas RS, Aarons G, Barg F, Evans A, Hadley T, Hoagwood K, et al. Policy to implementation: evidence-based practice in community mental health—study protocol. Implement Sci 2013;8(1):38.

6. Department of Veterans Affairs. Local implementation of evidence-based psychotherapies for mental and behavioral health conditions. 2012.

7. Smith TL, Landes SJ, Lester-williams K, Day KT, Batdorf W, Brown GK, et al. Developing alternative training delivery methods to improve psychotherapy implementation in the U.S. Department of Veterans Affairs. Train Educ Prof Psychol. 2017;11(4):266-75.

8. Rosen RC, Ruzek Jl, Karlin BE. Evidence-based training in the era of evidence-based practice: challenges and opportunities for training of PTSD providers. Behav Res Ther. 2017;88:37-48. 
9. Webb CA, DeRubeis RJ, Barber JP. Therapist adherence/competence and treatment outcome: a meta-analytic review. J Consult Clin Psychol. 2010; 78(2):200-11.

10. Farmer CC, Mitchell KS, Parker-Guilbert K, Galovski TE. Fidelity to the cognitive processing therapy protocol: evaluation of critical elements. Behav Ther. 2017:48(2):195-206.

11. Holder N, Holliday R, Williams R, Mullen K, Surís A. A preliminary examination of the role of psychotherapist fidelity on outcomes of cognitive processing therapy during an RCT for military sexual traumarelated PTSD. Cogn Behav Ther. 2018;47(1):76-89.

12. Proctor $E$, Silmere $H$, Raghavan $R$, Hovmand $P$, Aarons $G$, Bunger $A$, et al. Outcomes for implementation research: conceptual distinctions, measurement challenges, and research agenda. Adm Policy Ment Health Ment Health Serv Res. 2011;38(2):65-76

13. Stirman SW, Miller CJ, Toder K, Calloway A. Development of a framework and coding system for modifications and adaptations of evidence-based interventions. Implement Sci. 2013;8(1):65.

14. Powell BJ, Waltz TJ, Chinman MJ, Damschroder LJ, Smith JL, Matthieu MM, et al. A refined compilation of implementation strategies: results from the expert recommendations for implementing change (ERIC) project. Implement Sci. 2015;10(1):21.

15. Herschell AD, Kolko DJ, Baumann BL, Davis AC. The role of therapist training in the implementation of psychosocial treatments: a review and critique with recommendations. Clin Psychol Rev. 2010;30(4):448-66.

16. Beidas RS, Kendall PC. Training therapists in evidence-based practice: a critical review of studies from a systems-contextual perspective. Clin Psychol Publ Div Clin Psychol Am Psychol Assoc. 2010;17(1):1-30.

17. Jackson $C B$, Quetsch LB, Brabson LA, Herschell AD. Web-based training methods for behavioral health providers: a systematic review. Adm Policy Ment Health Ment Health Serv Res. 2018:45(4):587-610.

18. Whitlock EP, Green CA, Polen MR, Berg A, Klein J, Siu A, et al. Behavioral counseling interventions in primary care to reduce risky/harmful alcohol use [Internet]. Rockville (MD): agency for healthcare research and quality (US); 2004 [cited 2019 Feb 3]. (U.S. Preventive Services Task Force Evidence Syntheses, formerly Systematic Evidence Reviews). Available from: http:// www.ncbi.n/m.nih.gov/books/NBK42863/.

19. Moher D. Preferred reporting items for systematic reviews and metaanalyses: the PRISMA statement. Ann Intern Med. 2009;151(4):264.

20. Alberts $G$, Edelstein B. Therapist training: a critical review of skill training studies. Clin Psychol Rev. 1990;10(5):497-511.

21. Division 12, American Psychological Association. Research-Supported Psychological Treatments [Internet]. 2019. Available from: https://www.div12. org/psychological-treatments/.

22. Nathan PE, Gorman JM. A guide to treatments that work. 4th edition. Oxford University Press; 2015. https://www.oxfordclinicalpsych.com/view/1 0.1093/med:psych/9780199342211.001.0001/med-9780199342211.

23. Higgins JPT, Altman DG, Gøtzsche PC, Jüni P, Moher D, Oxman AD, et al. The Cochrane collaboration's tool for assessing risk of bias in randomised trials. BMJ. 2011;343:d5928.

24. Berkman ND, Lohr KN, Ansari MT, Balk EM, Kane R, McDonagh M, et al. Grading the strength of a body of evidence when assessing health care interventions: an EPC update. J Clin Epidemiol. 2015;68(11):1312-24.

25. Bearman SK, Schneiderman RL, Zoloth E. Building an evidence base for effective supervision practices: an analogue experiment of supervision to increase EBT fidelity. Adm Policy Ment Health Ment Health Serv Res. 2017:44(2):293-307.

26. Beidas RS, Edmunds JM, Marcus SC, Kendall PC. Training and consultation to promote implementation of an empirically supported treatment: a randomized trial. Psychiatr Serv. 2012;63(7):660-5.

27. Bennett-Levy J, Hawkins R, Perry H, Cromarty P, Mills J. Online cognitive behavioural therapy training for therapists: outcomes, acceptability, and impact of support: online CBT training. Aust Psychol. 2012;47(3):174-82.

28. Chu BC, Carpenter AL, Wyszynski CM, Conklin PH, Comer JS. Scalable options for extended skill building following didactic training in cognitivebehavioral therapy for anxious youth: a pilot randomized trial. J Clin Child Adolesc Psychol. 2017;46(3):401-10

29. Cohen JA, Mannarino AP, Jankowski K, Rosenberg S, Kodya S, Wolford GL. A randomized implementation study of trauma-focused cognitive behavioral therapy for adjudicated teens in residential treatment facilities. Child Maltreat. 2016;21(2):156-67.

30. Cooper Z, Bailey-Straebler S, Morgan KE, O'Connor ME, Caddy C, Hamadi L, et al. Using the internet to train therapists: randomized comparison of two scalable methods. J Med Internet Res [Internet]. 201718 [cited 2019 Jan 26] 19(10). Available from: https://www.ncbi.nlm.nih.gov/pmc/articles/ PMC5666223/.

31. Dimeff LA, Koerner K, Woodcock EA, Beadnell B, Brown MZ, Skutch JM, et al. Which training method works best? A randomized controlled trial comparing three methods of training clinicians in dialectical behavior therapy skills. Behav Res Ther. 2009;47(11):921-30.

32. Dimeff LA, Woodcock EA, Harned MS, Beadnell B. Can dialectical behavior therapy be learned in highly structured learning environments? Results from a randomized controlled dissemination trial. Behav Ther. 2011;42(2):263-75.

33. Dimeff LA, Harned MS, Woodcock EA, Skutch JM, Koerner K, Linehan MM. Investigating bang for your training buck: a randomized controlled trial comparing three methods of training clinicians in two core strategies of dialectical behavior therapy. Behav Ther. 2015;46(3):283-95.

34. Fu SS, Roth C, Battaglia CT, Nelson DB, Farmer MM, Do T, et al. Training primary care clinicians in motivational interviewing: a comparison of two models. Patient Educ Couns. 2015;98(1):61-8.

35. Gega L, Norman IJ, Marks IM. Computer-aided vs. tutor-delivered teaching of exposure therapy for phobia/panic: randomized controlled trial with preregistration nursing students. Int J Nurs Stud. 2007:44(3):397-405.

36. Harned MS, Dimeff LA, Woodcock EA, Skutch JM. Overcoming barriers to disseminating exposure therapies for anxiety disorders: a pilot randomized controlled trial of training methods. J Anxiety Disord. 2011;25(2):155-63.

37. Harned MS, Dimeff LA, Woodcock EA, Kelly T, Zavertnik J, Contreras I, et al. Exposing clinicians to exposure: a randomized controlled dissemination trial of exposure therapy for anxiety disorders. Behav Ther. 2014;45(6):731-44.

38. Henggeler SW, Sheidow AJ, Cunningham PB, Donohue BC, Ford JD. Promoting the implementation of an evidence-based intervention for adolescent marijuana abuse in community settings: testing the use of intensive quality assurance. J Clin Child Adolesc Psychol. 2008;37(3):682-9.

39. Hubley S, Woodcock EA, Dimeff LA, Dimidjian S. Disseminating behavioural activation for depression via online training: preliminary steps. Behav Cogn Psychother. 2015:43(02):224-38.

40. Larson MJ, Amodeo M, LoCastro JS, Muroff J, Smith L, Gerstenberger E. Randomized trial of web-based training to promote counselor use of cognitive behavioral therapy skills in client sessions. Subst Abuse. 2013:34(2):179-87.

41. Martino S, Ball SA, Nich C, Canning-Ball M, Rounsaville BJ, Carroll KM. Teaching community program clinicians motivational interviewing using expert and train-the-trainer strategies: teaching clinicians motivational interviewing. Addiction. 2011;106(2):428-41.

42. McDonough M, Marks IM. Teaching medical students exposure therapy for phobia/panic — randomized, controlled comparison of face-to-face tutorial in small groups vs. solo computer instruction. Med Educ. 2002;36(5):412-7.

43. Miller WR, Yahne CE, Moyers TB, Martinez J, Pirritano M. A randomized trial of methods to help clinicians learn motivational interviewing. J Consult Clin Psychol. 2004;72(6):1050-62.

44. Monson CM, Shields N, Suvak MK, Lane JEM, Shnaider P, Landy MSH, et al. A randomized controlled effectiveness trial of training strategies in cognitive processing therapy for posttraumatic stress disorder: impact on patient outcomes. Behav Res Ther. 2018;110:31-40.

45. Moyers TB, Manuel JK, Wilson PG, Hendrickson SML, Talcott W, Durand P. A randomized trial investigating training in motivational interviewing for behavioral health providers. Behav Cogn Psychother [Internet]. 2008 Mar [cited 2019 Jan 26];36(02). Available from: http://www.journals.cambridge. org/abstract_S1352465807004055.

46. Puspitasari AJ, Kanter JW, Busch AM, Leonard R, Dunsiger S, Cahill S, et al. A randomized controlled trial of an online, modular, active learning training program for behavioral activation for depression. J Consult Clin Psychol. 2017;85(8):814-25.

47. Rakovshik SG, McManus F, Vazquez-Montes M, Muse K, Ougrin D. Is supervision necessary? Examining the effects of internet-based CBT training with and without supervision. J Consult Clin Psychol. 2016;84(3):191-9.

48. Rawson RA, Rataemane S, Rataemane L, Ntlhe N, Fox RS, McCuller J, et al. Dissemination and implementation of cognitive behavioral therapy for stimulant dependence: a randomized trial comparison of 3 approaches. Subst Abuse. 2013;34(2):108-17.

49. Ruzek Jl, Rosen RC, Garvert DW, Smith LD, Sears KC, Marceau L, et al. Online self-administered training of PTSD treatment providers in cognitive-behavioral intervention skills: results of a randomized controlled trial: web-training randomized trial for trauma providers. J Trauma Stress. 2014 Dec;27(6):703-11. 
50. Smith JL, Carpenter KM, Amrhein PC, Brooks AC, Levin D, Schreiber EA, et al. Training substance abuse clinicians in motivational interviewing using live supervision via teleconferencing. J Consult Clin Psychol. 2012;80(3):450-64.

51. Weingardt KR, Villafranca SW, Levin C. Technology-based training in cognitive behavioral therapy for substance abuse counselors. Subst Abuse. 2006;27(3):19-25.

52. Zatzick D, Donovan DM, Jurkovich G, Gentilello L, Dunn C, Russo J, et al. Disseminating alcohol screening and brief intervention at trauma centers: a policy-relevant cluster randomized effectiveness trial: trauma center alcohol SBl effectiveness trial. Addiction. 2014;109(5):754-65.

53. Forsetlund L, Bjørndal A, Rashidian A, Jamtvedt G, O'Brien MA, Wolf FM, et al. Continuing education meetings and workshops: effects on professional practice and health care outcomes. Cochrane Database Syst Rev [Internet]. 200915 [cited 2020 Apr 28];2009(2). Available from: https:// www.ncbi.nlm.nih.gov/pmc/articles/PMC7138253/.

54. Bernardy NC, Hamblen JL, Friedman MJ, Ruzek Jl, McFall ME. Implementation of a posttraumatic stress disorder mentoring program to improve treatment services. Psychol Trauma Theory Res Pract Policy. 2011; 3(3):292-9.

55. Rash CJ, DePhilippis D, McKay JR, Drapkin M, Petry NM. Training workshops positively impact beliefs about contingency management in a nationwide dissemination effort. J Subst Abus Treat. 2013:45(3):306-12.

56. Beidas RS, Becker-Haimes EM, Adams DR, Skriner L, Stewart RE, Wolk CB, et al. Feasibility and acceptability of two incentive-based implementation strategies for mental health therapists implementing cognitive-behavioral therapy: a pilot study to inform a randomized controlled trial. Implement Sci. 2017;12(1):148.

57. Sayer NA, Rosen CS, Bernardy NC, Cook JM, Orazem RJ, Chard KM, et al. Context matters: team and organizational factors associated with reach of evidence-based psychotherapies for PTSD in the veterans health administration. Adm Policy Ment Health Ment Health Serv Res. 2017;44(6):904-18

58. Hallgren KA, Dembe A, Pace BT, Imel ZE, Lee CM, Atkins DC. Variability in motivational interviewing adherence across sessions, providers, sites, and research contexts. J Subst Abus Treat. 2018;84:30-41.

\section{Publisher's Note}

Springer Nature remains neutral with regard to jurisdictional claims in published maps and institutional affiliations.

Ready to submit your research? Choose BMC and benefit from:

- fast, convenient online submission

- thorough peer review by experienced researchers in your field

- rapid publication on acceptance

- support for research data, including large and complex data types

- gold Open Access which fosters wider collaboration and increased citations

- maximum visibility for your research: over $100 \mathrm{M}$ website views per year

At $\mathrm{BMC}$, research is always in progress.

Learn more biomedcentral.com/submissions 